\title{
Macrophage migration inhibitory factor rescues mesenchymal stem cells from doxorubicin-induced senescence though the PI3K-Akt signaling pathway
}

\author{
WENZHENG XIA ${ }^{1}$ and MENG HOU ${ }^{2}$ \\ Departments of ${ }^{1}$ Neurosurgery and ${ }^{2}$ Radiation Oncology, The First Affiliated Hospital, \\ Wenzhou Medical University, Wenzhou, Zhejiang 325000, P.R. China
}

Received May 29, 2017; Accepted November 1, 2017

DOI: 10.3892/ijmm.2017.3282

\begin{abstract}
Doxorubicin (DOXO), an anthracycline antibiotic, is a commonly used anticancer drug. Despite its widespread usage, the therapeutic effects of DOXO are limited by its cardiotoxicity. Mesenchymal stem cell (MSC)-based therapies have had positive outcomes in the treatment of DOXO-induced cardiac damage; however, DOXO exerts toxic effects on MSCs, decreasing the effectiveness of MSC therapy. Macrophage migration inhibitory factor (MIF) promotes MSC survival and rejuvenation, and thus is a promising candidate to protect MSCs against DOXO-induced injury. The present study revealed that DOXO induced the senescence of MSCs, resulting in decreased proliferation, viability and paracrine effects. However, pretreatment with MIF improved the proliferation rate, viability, paracrine function, telomere length and telomerase activity of MSCs. Furthermore, the results indicated that the molecular mechanism underlying the anti-senescent function of MIF involved the phosphatidylinositol 3-kinase (PI3K)-RAC- $\alpha$ serine/threonine-protein kinase (Akt) signaling pathway, which MIF activated. In agreement with this finding, silencing Akt was identified to abolish the anti-senescent effect of MIF. In addition, MIF decreased oxidative stress in MSCs, as revealed by the decreased production of reactive oxygen species and malondialdehyde, and the increased activity of superoxide dismutase. These results indicate that MIF can rescue MSCs from a state of DOXO-induced senescence by inhibiting oxidative stress and activating the PI3K-Akt signaling pathway. Thus, treatment with MIF may have an important therapeutic application for the rejuvenation of MSCs in patients with cancer being treated with DOXO.
\end{abstract}

Correspondence to: Dr Meng Hou, Department of Radiation Oncology, The First Affiliated Hospital, Wenzhou Medical University, 2 Fuxue Lane, Wenzhou, Zhejiang 325000, P.R. China E-mail: 244517813@qq.com

Key words: doxorubicin, mesenchymal stem cells, senescence, phosphatidylinositol 3-kinase/Akt signaling pathway, oxidative stress

\section{Introduction}

Doxorubicin (DOXO) is a widely used chemotherapeutic agent that is effective in treating hematological and solid tumor malignancies, including leukemia, breast cancer, lung carcinoma and kidney cancer (1). Despite its high efficacy, the clinical application of DOXO has been greatly restricted due to its side effects (2). Among the adverse effects, the major limiting factor for therapy with DOXO is cardiotoxicity, which is characterized by dilated cardiomyopathy that can develop for up to several years following cessation of treatment $(3,4)$. Thus, cardiotoxicity dramatically limits the use of DOXO as a chemotherapeutic agent.

Cell-based therapies have huge potential for treating DOXO-induced cardiotoxicity. Mesenchymal stem cell (MSC) transplantation is regarded as a promising option due to its regenerative effects and immunological safety $(5,6)$. However, DOXO has toxic effects on cultured MSCs, resulting in decreased proliferation and an impaired capacity for differentiation $(5,6)$. Furthermore, DOXO reduces the survival, induces the apoptosis and impairs the paracrine function of MSCs (7), indicating the induction of cellular senescence (8). Therefore, rejuvenating the activity of MSCs in the presence of DOXO could improve their therapeutic potential for the treatment of DOXO-induced cardiotoxicity.

Macrophage migration inhibitory factor (MIF) is a pleiotropic cytokine that maintains homeostasis by regulating physiological signaling pathways (9). MIF is thought to serve a fundamental role in cellular senescence; a previous study reported that in aged hearts, MIF secretion was significantly reduced and resulted in the dysregulation of glucose uptake during ischemia/reperfusion (10). In addition, it has been demonstrated that increasing the activity of MIF attenuates ischemia/reperfusion-induced injuries (11). Furthermore, MIF contributes to cell survival and proliferation, and has been identified to prevent cellular senescence (12). With respect to MSCs, MIF is a potential candidate for preventing naturally-occurring and hypoxia-induced senescence $(13,14)$. The present study investigated whether MIF could rejuvenate MSCs in the presence of DOXO and thus enhance their function. MIF could then be applied to rejuvenate MSCs and reduce DOXO-associated cardiomyopathy. 
Previous study has demonstrated that MIF acts through the phosphatidylinositol 3-kinase (PI3K)-RAC- $\alpha$ serine/threonineprotein kinase (Akt) signaling pathway to promote cellular resistance to glucose deprivation, ischemia, hypoxia, oxidative and senescence $(13,15)$. Activation of the PI3K-Akt signaling pathway can slow down the process of senescence, and has been investigated in MSCs as a therapeutic target forage-associated diseases $(12,13)$. Conversely, the downregulation of Akt expression can accelerate cellular senescence in MSCs (16). DOXO has been identified to impair Akt expression, leading to the senescence of human cardiac progenitor cells (17). In the present study, whether the activation of the PI3K-Akt signaling pathway by MIF could restore the proliferation and function of senescent MSCs in the presence of DOXO was investigated.

Cellular senescence can be triggered by multiple mechanisms, including those resulting in the production of reactive oxygen species (ROS) and oxidative stress (18). DOXO induces cellular injury primarily through the generation of ROS, which are a common mediator of cellular senescence $(19,20)$. It has also been demonstrated that MIF exerts a protective effect on MSCs by inhibiting ROS generation (15). Given the involvement of ROS in the senescence of MSCs (21), the present study hypothesized that modulating oxidative stress would rejuvenate senescent MSCs. Therefore, the present study investigated whether MIF could attenuate oxidative stress and promote the rejuvenation of senescent MSCs induced by DOXO.

\section{Materials and methods}

Animals. A total of 96 male Sprague Dawley rats (eight groups with $n=12 /$ group) weighing 60-80 g (age, 105.25 \pm 11.94 days) were purchased from the Laboratory Animals Center of Wenzhou Medical University (Wenzhou, China). The rats were cared for in accordance with published guidelines from the US National Institutes of Health (Bethesda, MD, USA) (22). The rats were raised apart and kept at a temperature of $21 \pm 2^{\circ} \mathrm{C}$, with a relative humidity of $30-70 \%$ and a 12 -h light/dark cycle. The rats had access to food and water ad libitum. All study procedures were approved by the Wenzhou Medical University Institutional Animal Care and Use Committee (Wenzhou, China).

Reagents. Dulbecco's modified Eagle's medium (DMEM) and fetal bovine serum (FBS) were purchased from HyClone (GE Healthcare Life Sciences, Logan,UT,USA). TRIzol reagent was obtained from Thermo Fisher Scientific, Inc. (Waltham, MA, USA). Rabbit monoclonal anti-rat antibodies directed against Akt (cat. no. 9272), phosphorylated (p)-Akt (S473, cat. no. 4060; T308, cat. no. 13038) and $\beta$-actin (cat. no. 4970) were purchased from Cell Signaling Technology, Inc. (Danvers, MA, USA) and the horseradish peroxidase-conjugated antirabbit secondary antibody (cat. no. sc-2357) was obtained from Santa Cruz Biotechnology, Inc. (Dallas, TX, USA). Sigma-Aldrich (Merck KGaA, Darmstadt, Germany) provided the enzyme-linked immunosorbent assay (ELISA) kits for vascular endothelial growth factor (VEGF; cat. no. RAB0512), basic fibroblast growth factor (bFGF; cat. no. RAB1139), hepatocyte growth factor (HGF; cat. no. RAB1145) and insulin-like growth factor (IGF; cat. no. RAB1146), and the MTT and dimethyl sulfoxide (DMSO). The small interfering (si)RNAs targeting Akt transcripts were prepared by Thermo Fisher
Scientific, Inc. Cell proliferation was assessed using the Cell Counting Kit-8 (CCK-8; HaiGene Technology, Harbin, China) assay. Rat recombinant MIF was obtained from Propec-Tany TechnoGene, Ltd. (East Brunswick, NJ, USA).

Cell culture and treatment. Bone marrow-derived MSCs were isolated and identified using a standard protocol as previously described (23). Briefly, MSCs were isolated from the bone marrow of the Sprague Dawley rats and cultured in DMEM supplemented with $10 \% \mathrm{FBS}$ at $37^{\circ} \mathrm{C}$ in $5 \% \mathrm{CO}_{2}$. The culture medium was changed every 2-3 days. All experiments were performed using MSCs from the third passage. The MSCs were pretreated with DOXO (5 $\mu \mathrm{mol} / \mathrm{l}$; Sigma-Aldrich; Merck KGaA) for $1 \mathrm{~h}$ at $37^{\circ} \mathrm{C}$, as previously described (7). Prior to subsequent tests, the MSCs were washed with phosphate-buffered saline (PBS). The concentration of treatment with MIF was $100 \mathrm{ng} / \mathrm{ml}$, and treatment was for $1 \mathrm{~h}$ at $37^{\circ} \mathrm{C}$.

Reverse transcription-quantitative polymerase chain reaction (RT-qPCR) analysis. Gene expression levels were analyzed by RT-qPCR. Briefly, total cellular RNA was isolated using TRIzol reagent and reverse transcribed using the Transcriptor First Stand cDNA Synthesis kit (Roche Applied Science, Mannheim, Germany) according to the manufacturer's protocol. qPCR was performed using the Fast Start Universal SYBR Master (Sigma-Aldrich; Merck KGaA) according to the manufacturer's protocol. The thermocycling conditions were as follows: 40 cycles of amplification at $95^{\circ} \mathrm{C}$ for $15 \mathrm{sec}$, followed by $64^{\circ} \mathrm{C}$ for $20 \mathrm{sec}$ and $72^{\circ} \mathrm{C}$ for $25 \mathrm{sec}$. The threshold number of cycles $(\mathrm{Cq})$ was set within the exponential phase of the reaction, and the $\Delta \mathrm{Cq}$ value for each target gene was calculated by subtracting the $\mathrm{Cq}$ value for the internal control, glyceraldehyde 3-phosphate dehydrogenase (GAPDH), from that of the target gene. Relative gene expression levels were calculated by comparing the $\Delta \mathrm{Cq}$ values between the control and experimental conditions for each target using the following equation: Relative gene expression $=2^{-(\Delta \mathrm{Cq} \text { sample- } \Delta \mathrm{Cq} \text { control })}(24)$. The primer pairs used to detect the mRNA levels of target genes are listed in Table I.

Cell proliferation assay. The rate of cell proliferation was estimated using a CCK-8 assay according to the manufacturer's protocol. Briefly, $1 \times 10^{4} \mathrm{MSC} /$ well were cultured in 96-well plates. When the cells reached $80-90 \%$ confluence, they were incubated with CCK-8 solution for $1 \mathrm{~h}$ at $37^{\circ} \mathrm{C}$, after which the absorbance of each well at $450 \mathrm{~nm}$ was recorded. The MSCs were treated with DOXO (5 $\mu \mathrm{mol} / \mathrm{l} /$ day $)$ and the proliferation rate was measured each day for the following 7 days.

MTT assay. The MTT assay was used to determine cell viability. A total of $1 \times 10^{4} \mathrm{MSC}$ /well were cultured in 96-well plates. When the cells reached $80-90 \%$ confluence, they were treated with DOXO $(5 \mu \mathrm{mol} / \mathrm{l})$ for $24 \mathrm{~h}$ at $37^{\circ} \mathrm{C}$. Then, a total of $300 \mu \mathrm{l}$ of MTT reagent was added to each well $2 \mathrm{~h}$ prior to harvesting. The supernatant was removed and the cells were incubated with $400 \mu \mathrm{l}$ of DMSO for $10 \mathrm{~min}$. The absorbance of the wells at $540 \mathrm{~nm}$ was recorded using a microplate reader.

ELISAs. The concentrations of MIF, VEGF, bFGF, HGF and IGF secreted by MSCs were assessed by ELISA according to 
Table I. Sequences of the primers used for reverse transcription-quantitative polymerase chain reaction analysis.

Primer (5'-3')

\begin{tabular}{lll}
\cline { 2 - 3 } Gene & \multicolumn{1}{c}{ Forward } & \multicolumn{1}{c}{ Reverse } \\
\hline MIF & ATGAACTTTCTGCTGTCTTG & TCACCGCCTCGGCTTGTCA \\
Telomere length & GGTTTTGAGGGTGAGGGTGAGGGTGAGGGTGA & TCCCGACTATCCCTATCCCTATCCCTATCCCTATCC \\
Akt & TCGTGTGGCAAGATGTGTATGAGA & CAGGCGCGTGTGGTGAT \\
p53 & TCTGTCATCTTCCGTCCCTTCTC & CCGTGCACATAACAGACTTGGCT \\
p16 & GGTCACCGACAGGCATAACTTC & AAAGGAGGGCTGAGGCCTAA \\
GAPDH & GGCTCTCTGCTCCTCCCTGTT & GGCTCTCTGCTCCTCCCTGTT \\
\hline
\end{tabular}

MIF, macrophage migration inhibitory factor; Akt, RAC- $\alpha$ serine/threonine-protein kinase; GAPDH, glyceraldehyde 3-phosphate dehydrogenase.

the manufacturer's protocol, as previously described (15). The absorbance of each well was quantified at $450 \mathrm{~nm}$.

ROS measurement. Levels of intracellular ROS were determined using 2,7-dichlorodihydrofluorescein diacetate (Beyotime Institute of Biotechnology, Haimen, China), following the manufacturer's protocol. The fluorescent intensity of the cells was measured using a fluorescence spectrophotometer, with excitation and emission wavelengths of 488 and $525 \mathrm{~nm}$, respectively.

Superoxide dismutase (SOD) activity assay. SOD activity in the MSCs was determined using a colorimetric assay kit (SOD activity assay kit; cat. no. ab65354; Abcam, Cambridge, UK) according to the manufacturer's protocol. Briefly, protein was isolated from MSCs using cell lysis buffer (Beyotime Institute of Biotechnology) and SOD activity was measured in $10 \mu \mathrm{g}$ of total protein extract. Absorbance was measured at $450 \mathrm{~nm}$.

Lipid peroxidation assay. Lipid peroxidation was monitored using a Lipid Peroxidation (MDA) assay kit (Colorimetric/Fluorometric; Abcam) to measure the formation of malondialdehyde (MDA) according to the manufacturer's protocol. Briefly, MSCs $\left(1 \times 10^{6}\right.$ cells) were homogenized on ice in $300 \mu \mathrm{l}$ of MDA lysis buffer (with $3 \mu \mathrm{l}$ of $100 \mathrm{X}$ butylated hydroxytoluene), then centrifuged $(13,000 \mathrm{x}$ g for $10 \mathrm{~min}$ at $\left.4^{\circ} \mathrm{C}\right)$ to remove insoluble material. The supernatant $(200 \mu \mathrm{l})$ was added to $600 \mu \mathrm{l}$ of thiobarbituric acid and incubated at $95^{\circ} \mathrm{C}$ for $60 \mathrm{~min}$. The samples were then cooled to room temperature in an ice bath for $10 \mathrm{~min}$ and their absorbance at $532 \mathrm{~nm}$ was measured spectrophotometrically.

Relative telomere length measurement. Quantification of the relative telomere length in MSCs was performed using qPCR based on a previously established method (25), using the aforementioned RT-qPCR protocol and GAPDH as the normalizing gene. The primer pairs used to detect telomere length were as follows: Forward, 5'-GGTTTTTGAGGGTGAGGGTGAGGG TGAGGGTGA-3' and reverse, 5'-TCCCGACTATCCCTATCC CTATCCCTATCCCTATCC-3'

Relative telomerase activity measurement. The telomerase activity of MSCs was examined using the TeloTAGGG
Telomerase PCR ELISAPLUS kit (Sigma-Aldrich; Merck KGaA) according to the manufacturer's protocol. Cell lysis buffer provided in the kit was used to lyse the MSCs. After incubation for $30 \mathrm{~min}$ on ice, cell lysates were centrifuged for $20 \mathrm{~min}$ at $4^{\circ} \mathrm{C}(12,000 \mathrm{x} \mathrm{g})$. A total of $3 \mu \mathrm{g}$ of cell extract was used for each telomeric repeat amplification reaction and $3 \mu \mathrm{l}$ of inactivated cell lysate was used for telomeric repeat amplification protocol (TRAP) reactions. Each TRAP reaction was performed with amplification of an internal control from the kit. The amplified products were immobilized on streptavidin-coated microtiter plates via biotin-streptavidin interaction. Subsequently, amplification was detected by adding $100 \mu \mathrm{l}$ anti-digoxigenin antibodies conjugated to horseradish peroxidase (provided in the kit) to each well, and incubating the plate at $15-25^{\circ} \mathrm{C}$ for $30 \mathrm{~min}$ while shaking at $300 \mathrm{rpm}$. After the addition of the peroxidase substrate (3,3',5,5'-tetramethylbenzidine), the amount of TRAP product was determined by measuring the absorbance at $450 \mathrm{~nm}$ using a microplate reader.

Western blot analysis. Western blotting was performed as previously described (15). Briefly, cells were washed twice with ice-cold PBS and lysed with cell lysis buffer [20 mM Tris ( $\mathrm{pH} 7.5$ ), $150 \mathrm{mM} \mathrm{NaCl}, 1 \%$ Triton $\mathrm{X}-100$, sodium pyrophosphate, $\beta$-glycerophosphate, EDTA, $\mathrm{Na}_{3} \mathrm{VO}_{4}$ and leupeptin; Beyotime Institute of Biotechnology]. The lysates were centrifuged for $5 \mathrm{~min}$ at $12,000 \times \mathrm{g}\left(4^{\circ} \mathrm{C}\right)$ and the resulting supernatant contained the total cellular protein. A BCA assay was used for protein quantification. For each sample, $20 \mu \mathrm{g}$ total protein/lane was resolved by $5-10 \%$ sodium dodecyl sulfate-polyacrylamide gel electrophoresis (SDS-PAGE) and transferred onto polyvinylidene difluoride membranes. The membranes were blocked for $1 \mathrm{~h}$ at $37^{\circ} \mathrm{C}$ with 5\% skimmed milk in Tris-buffered saline containing $0.1 \%$ Tween-20, and then incubated overnight at $4^{\circ} \mathrm{C}$ with the primary antibodies directed against Akt, p-Akt and $\beta$-actin $(1: 1,000)$. The membranes were washed, and then incubated for $1 \mathrm{~h}$ at $37^{\circ} \mathrm{C}$ with the horseradish peroxidase-conjugated secondary antibodies and developed using chemiluminescent substrate (BeyoECL Plus; Beyotime Institute of Biotechnology). The stained protein bands were visualized using a ChemiDoc XRS system and quantified using Quantity One software v. 4.5.2 (both from Bio-Rad Laboratories, Inc., Hercules, CA, USA). 
A

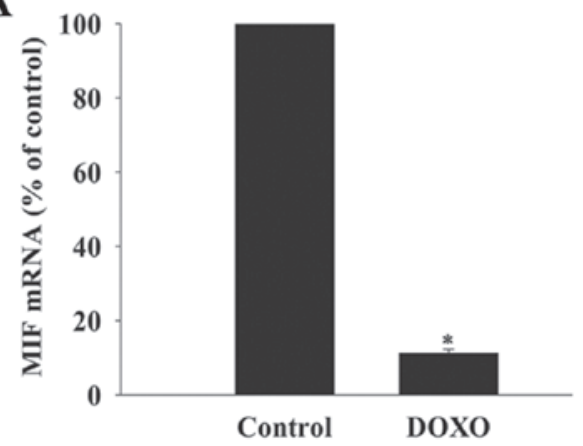

B

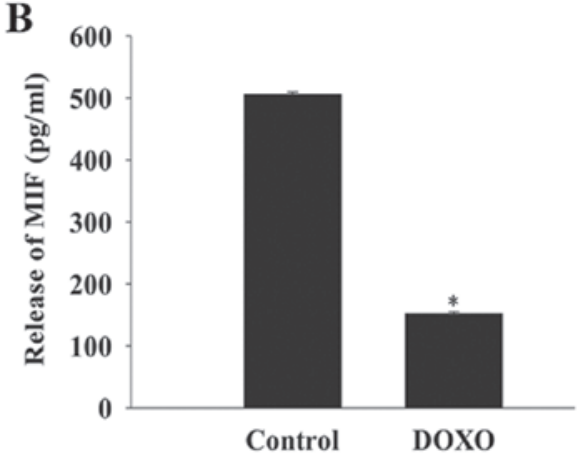

Figure 1. DOXO decreases the expression and release of MIF in MSCs. (A) Reverse transcription-quantitative polymerase chain reaction analysis of MIF mRNA levels in MSCs with and without DOXO treatment. (B) The concentration of MIF in the culture medium of MSCs and DOXO-treated MSCs was analyzed by ELISA. ${ }^{*} \mathrm{P}<0.05$ vs. the control group. DOXO, doxorubicin; MSC, mesenchymal stem cell; MIF, macrophage migration inhibitory factor; ELISA, enzyme-linked immunosorbent assay.

Knockdown of gene expression using siRNA. MSCs were transfected using X-tremeGENE HP DNA Transfection reagent (Roche Life Sciences) according to the manufacturer's protocol. Briefly, MSCs (1x10 cells/well) were cultured in 6-well plates for $24 \mathrm{~h}$, then treated with the transfection reagent at a reagentto-siRNA weight ratio of $3: 1$ for $20 \mathrm{~min}$, followed by the addition of a mixture containing $100 \mathrm{nM}$ siRNA and incubation in $2 \mathrm{ml}$ DMEM for $48 \mathrm{~h}$ at $37^{\circ} \mathrm{C}$. Scrambled non-targeting siRNA (siRNA-NT) was used as a negative control. The efficiency of Akt knockdown was determined by RT-qPCR, as described above. The sequences of the siRNAs used were as follows: siRNA-Akt, GAUCUCCUCAUCAUCUGGATT; and siRNA-NT, UUCUCCGAACGUGUCACGUTT.

Statistical analysis. Data were expressed as the mean \pm standard deviation from three independent experiments. Differences among groups were tested by one-way analysis of variance followed by Tukey's multiple comparisons test. Comparisons between two groups were evaluated using the paired Student's t-test. All statistical analyses were performed using SPSS software (version 19.0; IBM Corp., Armonk, NY, USA). $\mathrm{P}<0.05$ was considered to indicate a statistically significant difference.

\section{Results}

DOXO decreases the expression and release of MIF in MSCs. The basal mRNA expression and release of MIF from MSCs was measured after exposure to DOXO at a concentration of $5 \mu \mathrm{mol} / \mathrm{l}$ using RT-qPCR analysis and an ELISA. This revealed that the baseline expression of MIF mRNA and release of MIF from cells exposed to DOXO was significantly decreased compared with the control group (Fig. 1).

DOXO induces the senescence of MSCs. DOXO has been demonstrated to be cytotoxic to MSCs (5), so whether DOXO could induce the senescence of MSCs was investigated in the present study. When MSCs were treated with DOXO at a concentration of $5 \mu \mathrm{mol} / 1$, the proliferation rate decreased significantly, starting immediately after treatment and lasting for 7 days (Fig. 2A). Furthermore, DOXO treatment significantly decreased MSC viability compared with the control group, as measured by the MTT assay (Fig. 2B).
MSCs secrete a variety of cytokines and growth factors that can function in a paracrine and autocrine manner, and their trophic effects on MSCs are known to be impaired by senescence (14). As expected, the release of VEGF, bFGF, HGF and IGF was significantly lower in MSCs treated with $5 \mu \mathrm{mol} / 1 \mathrm{DOXO}$ compared with the control group (Fig. 2C-F). Furthermore, the expression of the senescence-associated genes cellular tumor antigen p53 and p16 was significantly increased in the DOXO treatment group compared with the control group (Fig. 2G and H).

Exogenous MIF rescues MSCs from DOXO-induced senescence. A previous study has revealed that exogenous MIF (100 ng/ml) increases the survival and rejuvenation of MSCs (15). The present study identified that $100 \mathrm{ng} / \mathrm{ml} \mathrm{MIF}$ pretreatment in the presence of DOXO significantly increased MSC proliferation (Fig. 3A) and cell viability (Fig. 3B) compared with the DOXO group. In addition, MIF increased trophic effects, as indicated by the significantly increased levels of secreted VEGF, bFGF, HGF and IGF compared with the group treated with DOXO alone (Fig. 3C-F). Furthermore, MIF treatment significantly increased telomere length and restored telomerase activity, which were decreased by exposure to DOXO (Fig. 3G and H).

MIF exerts its anti-senescent effect through the PI3K-Akt signaling pathway. The PI3K-Akt signaling pathway promotes survival and rejuvenation in numerous cell types (13). Therefore, whether this pathway mediated the anti-senescent effect of MIF in MSCs was investigated. Compared with the control group, treatment with DOXO at a concentration of $5 \mu \mathrm{mol} / 1$ significantly decreased the phosphorylation of Akt, which was restored by MIF (Fig. 4).

To further confirm the role of the PI3K-Akt pathway in the anti-senescent effect of MIF, Akt was silenced using siRNA and the senescence of the MSCs was examined in the presence of DOXO with or without MIF. The knockdown of Akt significantly decreased the expression of Akt compared with the control group (Fig. 5A). Silencing Akt significantly attenuated the anti-senescent effect of MIF, as demonstrated by the decreased proliferation (Fig. 5B), viability (Fig. 5C) and hormone secretion (Fig. 5D-G) of MSCs following the knockdown. Furthermore, silencing Akt significantly 

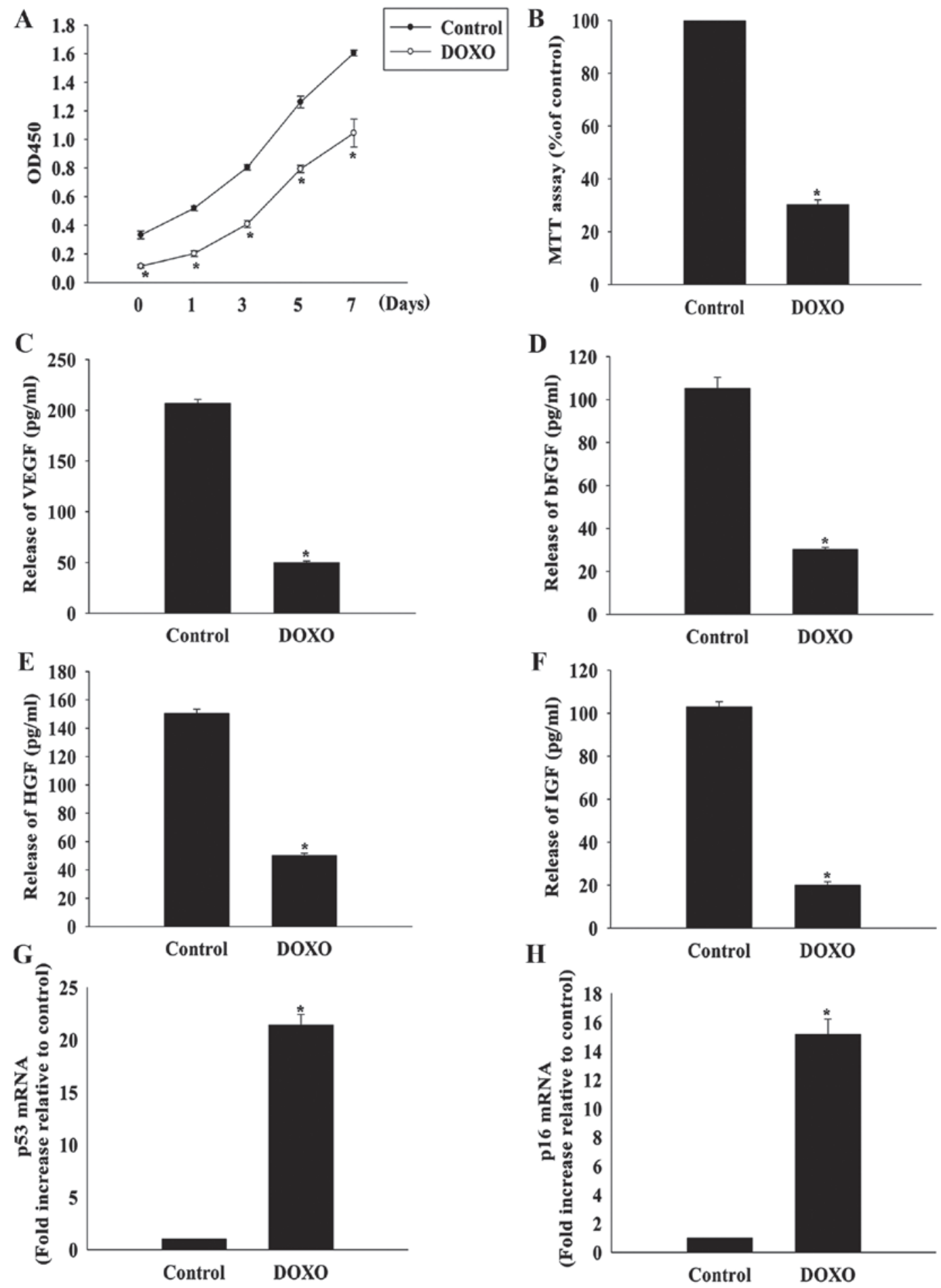

Figure 2. DOXO induces the senescence of MSCs. (A) Proliferation growth curves of MSCs incubated with or without DOXO were determined using the Cell Counting Kit-8 assay after 1,3,5 and 7 days of treatment. (B) MSC viability after DOXO treatment was analyzed using the MTT assay. The relative concentration of (C) VEGF, (D) bFGF, (E) HGF and (F) IGF was calculated using ELISAs in MSCs with or without DOXO treatment. Reverse transcription-quantitative polymerase chain reaction analysis of $(\mathrm{G}) \mathrm{p} 53$ and $(\mathrm{H}) \mathrm{p} 16 \mathrm{mRNA}$ levels in MSCs treated with DOXO. "P<0.05 vs. the control group. DOXO, doxorubicin; MSC, mesenchymal stem cell; VEGF, vascular endothelial growth factor; bFGF, basic fibroblast growth factor; HGF, hepatocyte growth factor; IGF, insulin-like growth factor; p53, cellular tumor antigen p53; ELISA, enzyme-linked immunosorbent assay.

shortened telomere length (Fig. 5H) and decreased telomerase activity (Fig. 5I). By contrast, siRNA-NT had no significant effect on any of these factors.

MIF exhibits anti-senescent effects via inhibiting oxidative stress. To determine whether the anti-senescent effect of MIF in the presence of DOXO involved the amelioration of oxidative stress, the generation of ROS, activation of SOD and lipid peroxidation were examined (Fig. 6). Compared with the control group, DOXO significantly increased the generation of ROS and MDA activation, while decreasing the activation of SOD. However, when treated with MIF, the generation of ROS and MDA was significantly decreased and the activation of SOD was significantly increased. siRNA 
$\mathbf{A}$

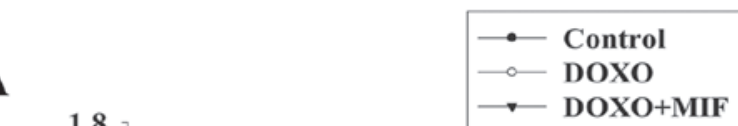

C
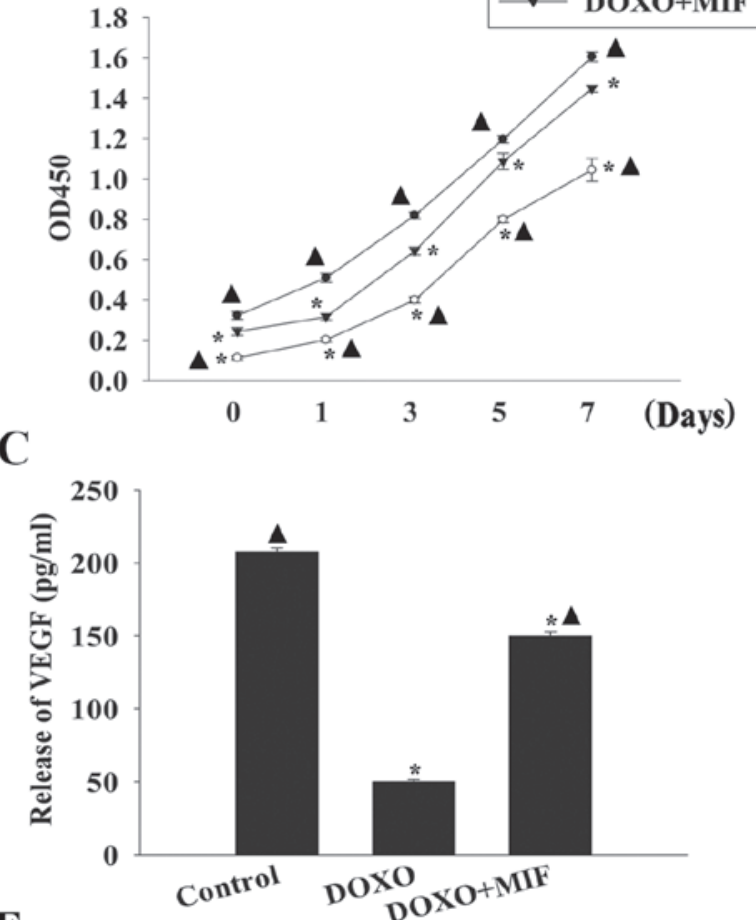

$\mathbf{E}$
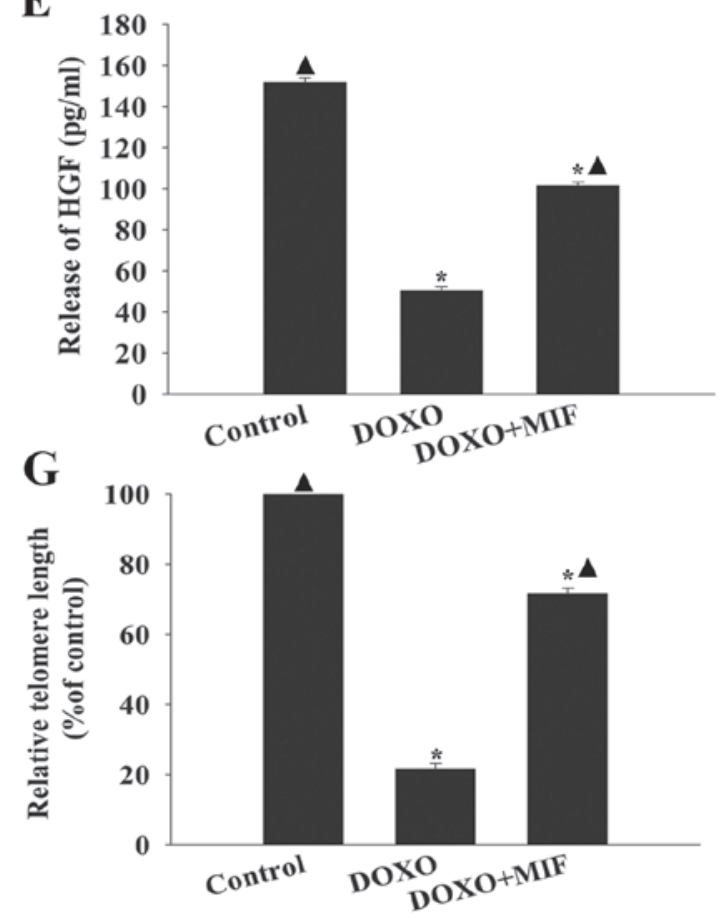

B

D
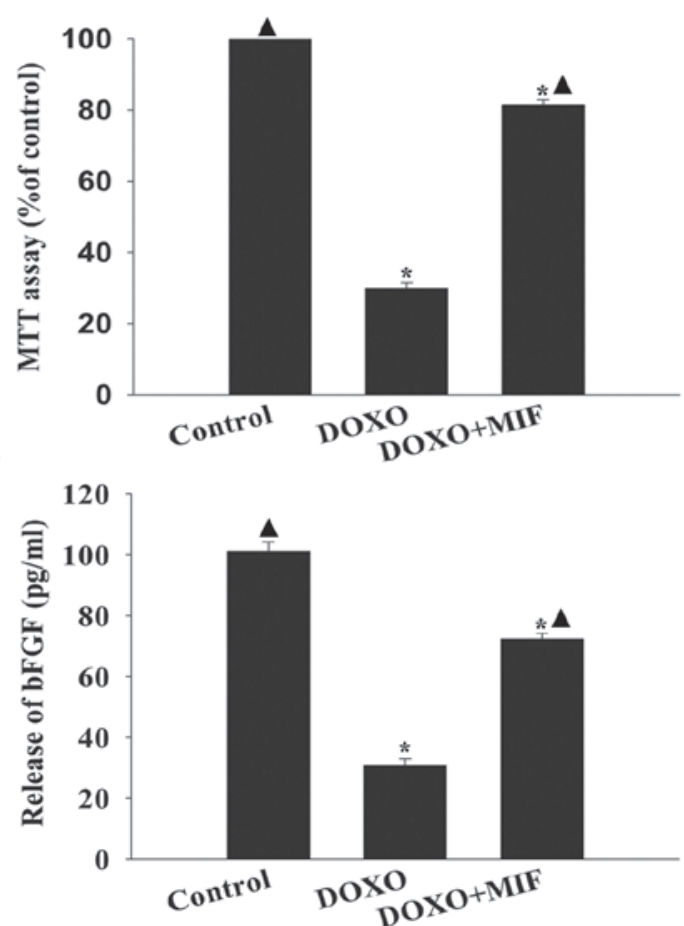

F

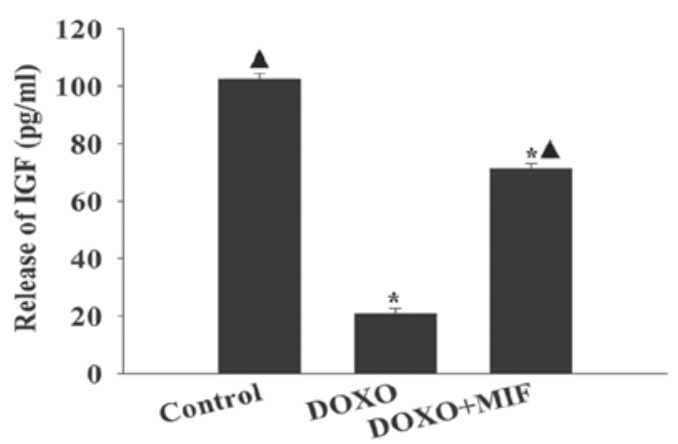

H

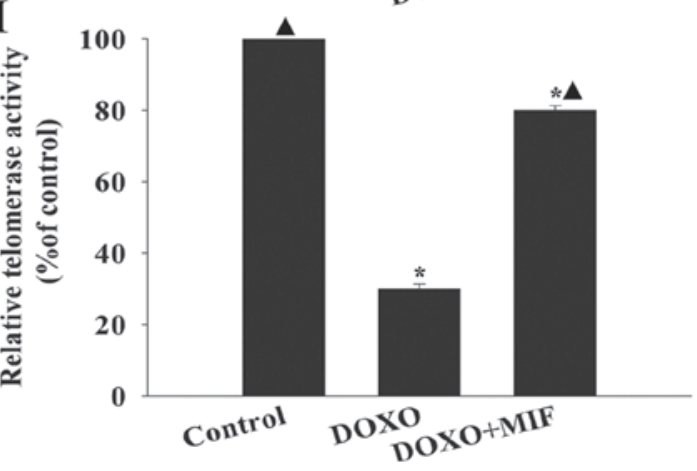

Figure 3. Exogenous MIF rescues MSCs from DOXO-induced senescence. (A) Proliferation growth curves for untreated, DOXO-treated and DOXO+MIF-treated MSCs were determined using the Cell Counting Kit-8 assay. (B) MSC viability was analyzed using the MTT assay in untreated MSCs, MSCs treated with DOXO and MSCs treated with MIF in presence of DOXO. The relative concentration of (C) VEGF, (D) bFGF, (E) HGF and (F) IGF was calculated using ELISAs. (G) Analysis of telomere length through mRNA levels was performed using reverse transcription-quantitative polymerase chain reaction analysis. $(\mathrm{H})$ Relative telomerase activity was measured using the telomeric repeat amplification protocol assay. ${ }^{*} \mathrm{P}<0.05$ vs. the control group; ${ }^{\wedge} \mathrm{P}<0.05$ vs. the $\mathrm{DOXO}$ group. MIF, macrophage migration inhibitory factor; MSC, mesenchymal stem cell; DOXO, doxorubicin; VEGF, vascular endothelial growth factor; bFGF, basic fibroblast growth factor; HGF, hepatocyte growth factor; IGF, insulin-like growth factor; OD, optical density; ELISA, enzyme-linked immunosorbent assay.

directed against Akt significantly blocked the inhibitory effect of MIF on oxidative stress, resulting in the increased generation of ROS and MDA, while decreasing the activation of SOD.

\section{Discussion}

Chemotherapy is an essential tool in cancer treatment; however, as the number of cancer survivors has increased, 

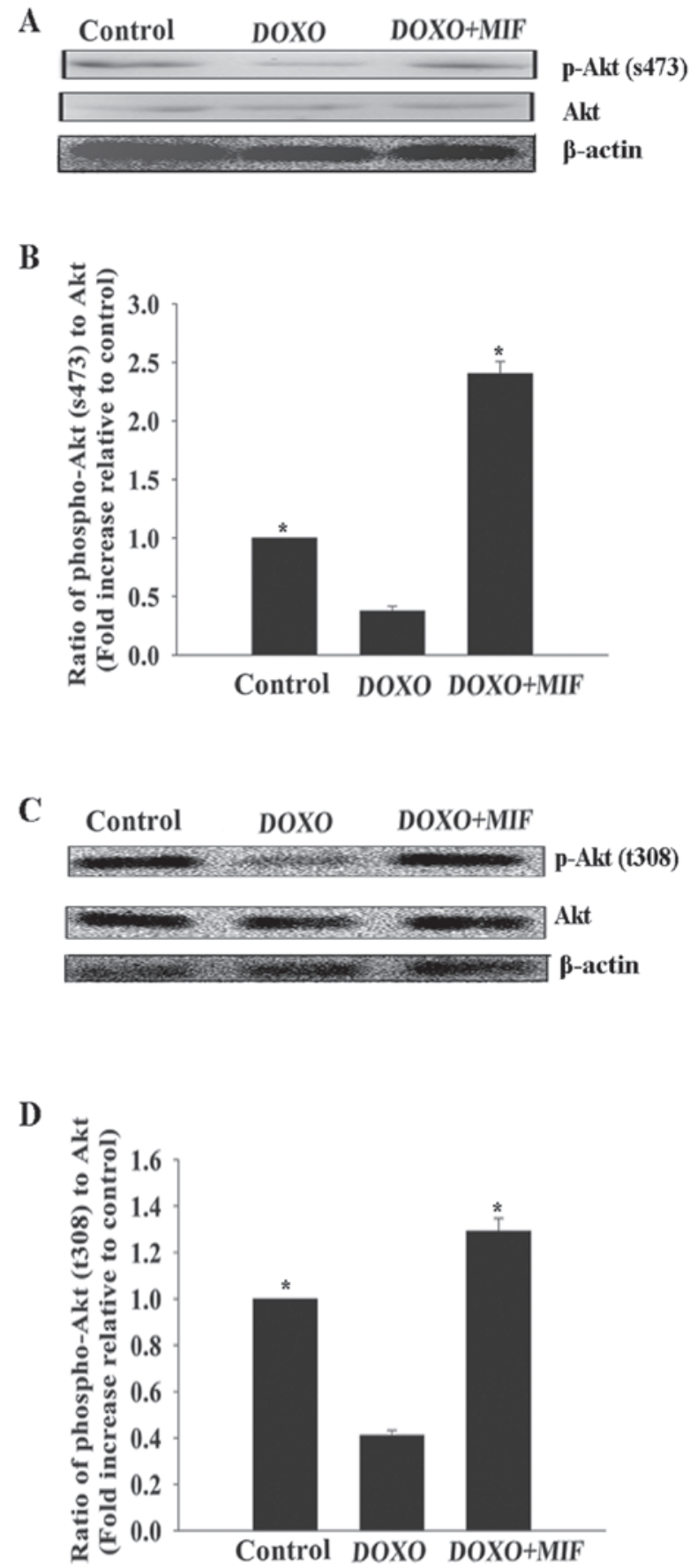

Figure 4. MIF induces activation of the phosphatidylinositol 3-kinase-Akt signaling pathway. (A) Representative western blotting and (B) quantification of Akt and p-Akt (s473) in untreated MSCs, MSCs treated with DOXO and MSCs treated with MIF in presence of DOXO. (C) Representative western blotting and (D) quantification of Akt and p-Akt (t308) in untreated, DOXO-treated and DOXO+MIF-treated MSCs. ${ }^{*} \mathrm{P}<0.05$ vs. the DOXO group. MIF, macrophage migration inhibitory factor; MSC, mesenchymal stem cell; DOXO, doxorubicin; p-, phosphorylated; Akt, RAC- $\alpha$ serine/threonine-protein kinase.

concerns about the effects of chemotherapy on the quality of life and lifespan of patients have been highlighted (26). DOXO, which belongs to the anthracycline family, has been proven to be an effective treatment for numerous types of cancer, including breast, lung, stomach, bladder and skin cancer. However, despite its high efficacy as a therapeutic agent against these tumors, DOXO is often accompanied by unavoidable adverse effects, such as cardiovascular toxicity $(27,28)$. The spectrum of short- and long-term cardiotoxic effects induced by DOXO ranges from cardiomyocyte senescence and subclinical ventricular dysfunction to severe cardiomyopathy and heart failure, necessitating cardiac transplantation and potentially resulting in mortality $(28,29)$. Several studies have revealed the potential mechanisms by which DOXO causes these effects, including the disruption of energy metabolism and apoptosis, increasing the production of ROS and inducing mitochondrial injury $(30,31)$. Therefore, enhancing the therapeutic effect of DOXO while decreasing its adverse cardiotoxic effects is an important objective $(32,33)$.

Among the strategies that have been investigated to prevent or repair cardiac injury, MSC transplantation is promising candidate due to the regenerative properties, paracrine function and immune modulatory effects of MSCs (6). A previous study reported that the intramuscular injection of MSCs improved cardiac function in a DOXO-induced dilated cardiomyopathy (DCM) rat model and attenuated DCM-associated mitochondria impairment (34). In addition, the transplantation of MSCs protects cardiomyocytes against DOXO-induced cardiomyopathy through paracrine effects (33). However, although MSCs exhibit therapeutic benefits against DOXO-induced cardiomyopathy, DOXO can cause MSC injury. It has been reported that DOXO impaired the proliferation, decreased the production of connexin 43 and hindered the capacity of MSCs to respond to cardiomyogenic differentiation stimuli (5). A previous study also revealed that DOXO induced MSC apoptosis and impaired the paracrine effects of MSCs (7). The present study identified that DOXO induced MSC senescence, rapidly resulting in a lower proliferation rate. This is in agreement with previous study, which revealed that the combination of DOXO and low intensity ultrasound significantly decreased the survival rate of C6 cells (35). Treatment with DOXO also impaired the viability and decreased the paracrine effects of MSCs in the present study, indicating that MSCs cannot inhibit DOXO-induced cardiomyopathy.

MIF is a proinflammatory cytokine, which was originally identified to serve an important role in chronic inflammatory diseases (36). Previous studies have suggested that MIF is involved in cell proliferation, survival and senescence $(9,14)$. In regards to senescence, it has been demonstrated that MIF expression is reduced in aged hearts (37). A previous study also revealed that cardiomyocytes in MIF-deficient mice exhibited contractile defects in response to starvation and underwent increased apoptosis during ischemia/reperfusion (10). Mechanisms underlying this MIF-dependent anti-senescent effect are associated with the modulation of senescence via signaling pathways, genes and enzymes (38). It has been demonstrated that MIF maintains neural stem cell properties, and promotes cell survival and proliferation (12). MIF also rejuvenates MSCs under hypoxic conditions (13). Previous study by our group demonstrated that MIF could rejuvenate aged MSCs, leading to increased proliferation rates, improved paracrine effects and an improved resistance to hypoxia/ischemia-induced apoptosis (14). A recent study revealed that overexpression of the MIF gene in induced pluripotent stem cell-derived MSCs improved DOXO-induced 
A

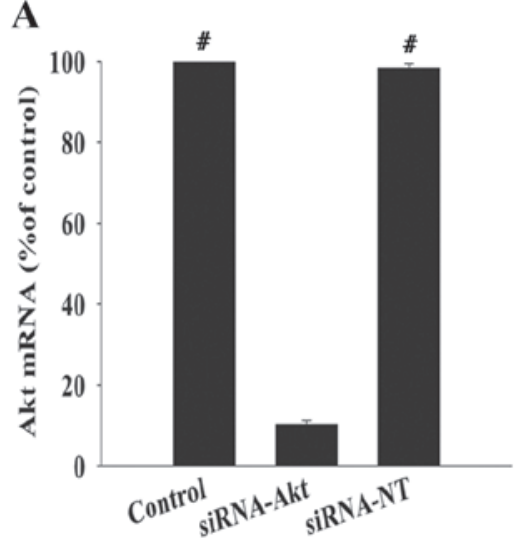

D

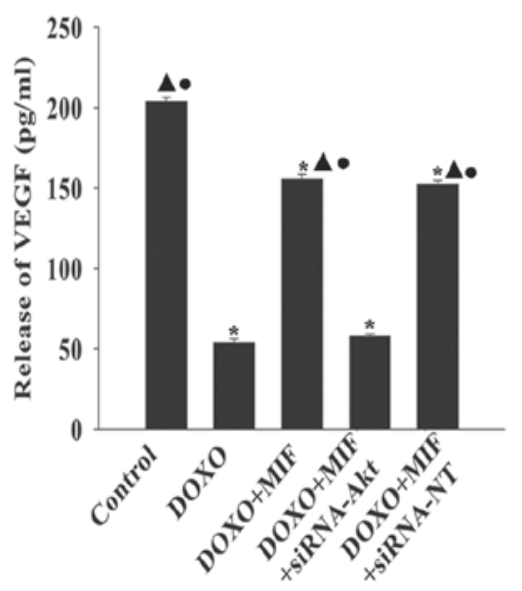

G

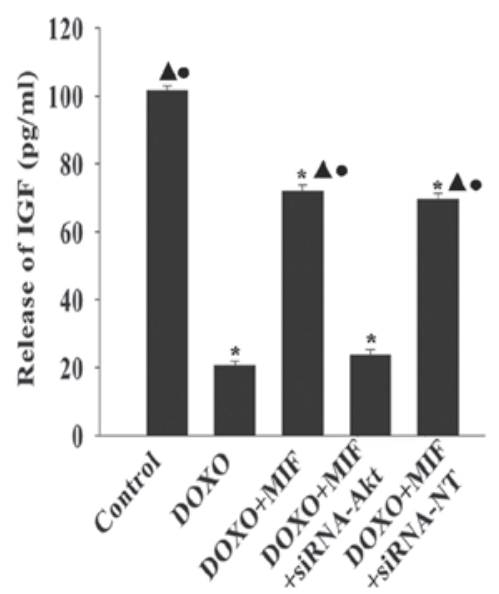

B

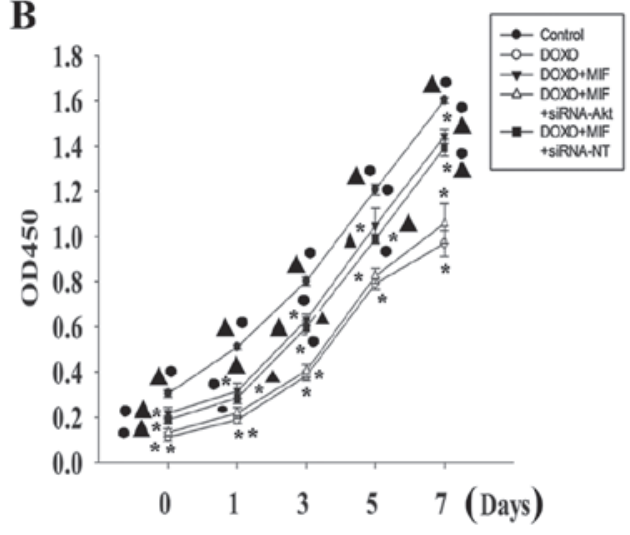

$\mathbf{E}$

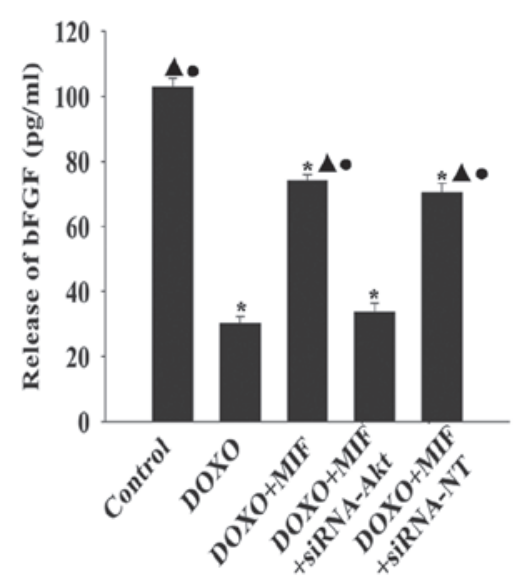

H

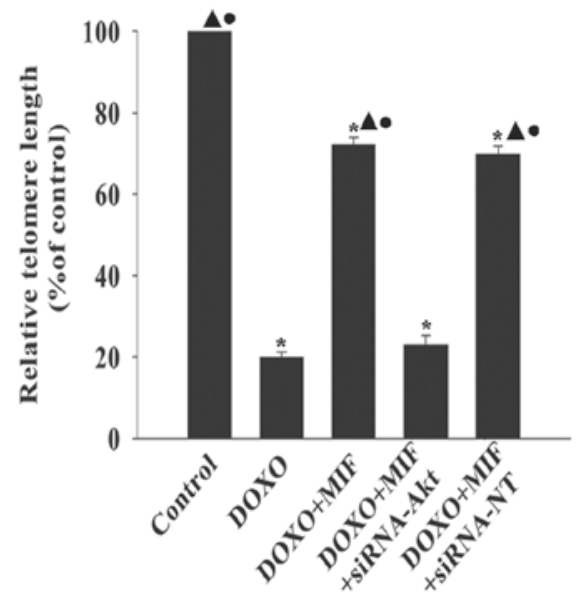

C
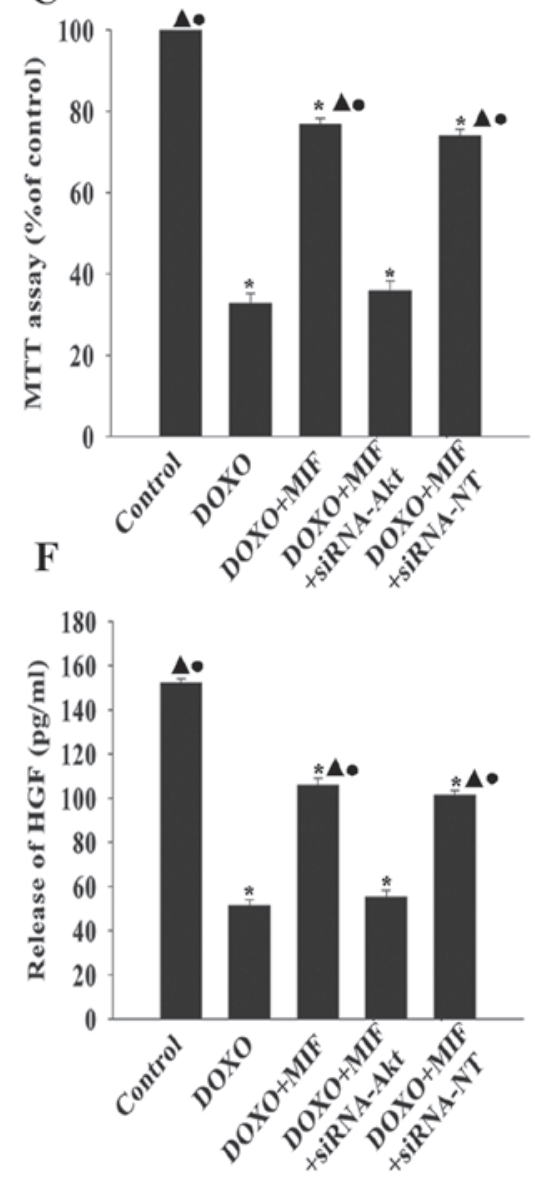

I

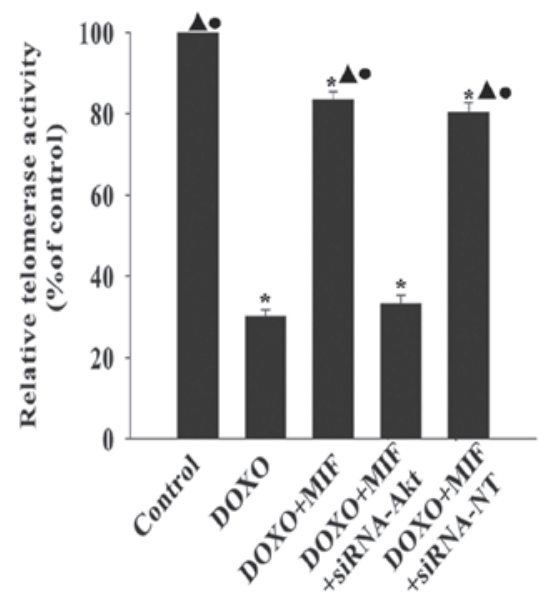

Figure 5. MIF exerts its anti-senescent effect through the phosphatidylinositol 3-kinase-Akt signaling pathway. (A) MSCs were transfected with siRNA directed against Akt or with siRNA-NT as a control, and the expression of Akt mRNA was determined by RT-qPCR. "P<0.05 vs. the siRNA-Akt group. To determine if Akt was involved in the anti-senescent effect of MIF, MSCs were transfected with siRNA directed against Akt or with siRNA-NT as a control, then treated with MIF in the presence of DOXO. (B) Proliferation growth curves of the MSCs were determined using the Cell Counting Kit-8 assay. (C) Cell viability was analyzed using the MTT assay. The relative concentration of (D) VEGF, (E) bFGF, (F) HGF and (G) IGF were analyzed using ELISAs. (H) Analysis of telomere length through mRNA levels was performed using RT-qPCR. (I) Relative telomerase activity was measured using the telomeric repeat amplification protocol assay. ${ }^{\prime} \mathrm{P}<0.05$ vs. the control group; ${ }^{\wedge} \mathrm{P}<0.05$ vs. the DOXO group. ${ }^{\bullet} \mathrm{P}<0.05$ vs. the DOXO+MIF+siRNA-Akt group. MIF, macrophage migration inhibitory factor; MSC, mesenchymal stem cell; DOXO, doxorubicin; p-, phosphorylated; Akt, RAC- $\alpha$ serine/threonine-protein kinase; si, small interfering; NT, non-targeting; RT-qPCR, reverse transcription-quantitative polymerase chain reaction; ELISA, enzyme-linked immunosorbent assay.

cardiomyopathy (33). The present study identified that in the presence of DOXO, pretreatment with MIF could drive MSCs into a rejuvenated state, in which the cells exhibited increased proliferation, viability and paracrine effects. Furthermore,
MIF increased telomere length and telomerase activity, which were impaired by DOXO treatment.

In addition to its role in regulating cell survival, proliferation and glucose metabolism, the PI3K-Akt signaling 

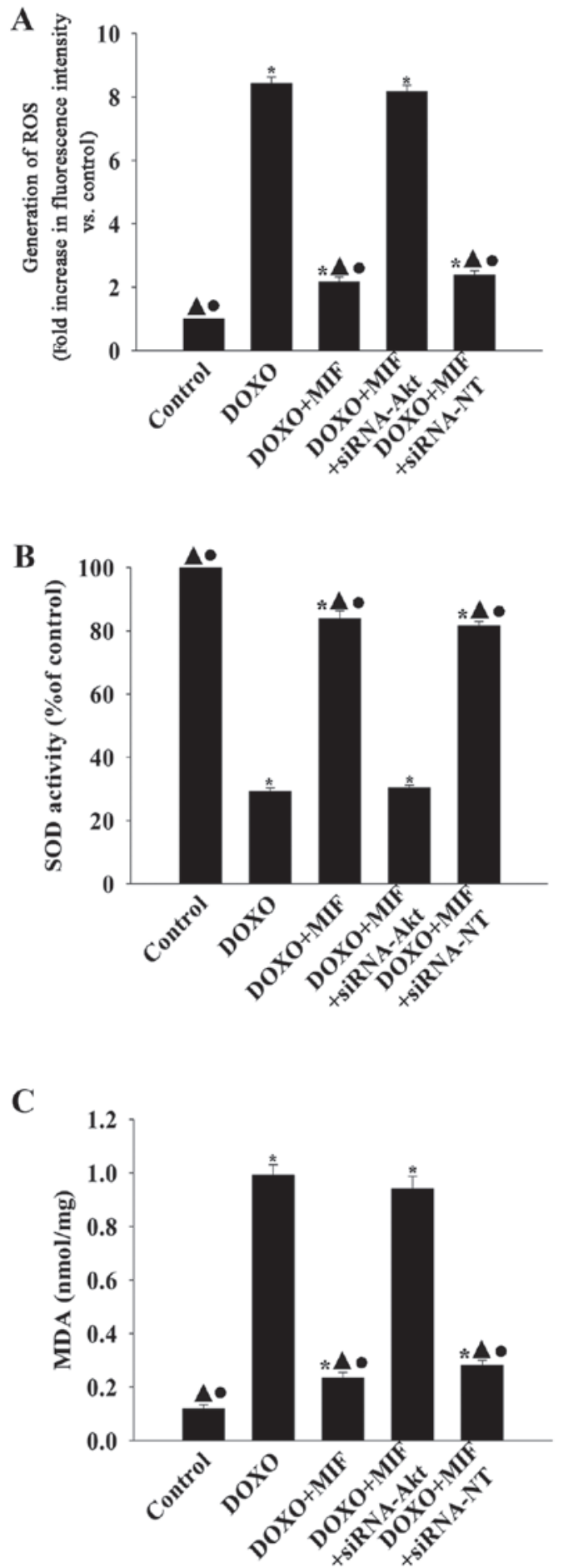

Figure 6. MIF exhibits anti-senescent effects via inhibiting oxidative stress. To determine if the modulation of oxidative stress was involved in the antisenescent effect of MIF, MSCs were transfected with siRNA directed against Akt or with siRNA-NT as a control. MSCs were then treated with MIF in the presence of DOXO. (A) The intracellular production of ROS in MSCs was analyzed using a fluorescence spectrophotometer. (B) SOD activity was evaluated using a colorimetric assay. (C) MDA formation was assayed to evaluate lipid peroxidation. ${ }^{*} \mathrm{P}<0.05$ vs. the control group; ${ }^{\wedge} \mathrm{P}<0.05$ vs. the DOXO group; $\bullet^{\bullet}<0.05$ vs. the DOXO+MIF+siRNA-Akt group. MIF, macrophage migration inhibitory factor; MSC, mesenchymal stem cell; DOXO, doxorubicin; si, small interfering; NT, non-targeting; ROS, reactive oxygen species; SOD, superoxide dismutase; MDA, malondialdehyde.

pathway regulates cellular senescence through the functional modulation of various gene products $(39,40)$. Akt is a stress- signaling kinase and key regulator of energy metabolism pathways that protect cells against stress-induced injury and death (13). DOXO has been revealed to impair this signaling pathway to induce cardiomyopathy (41). The use of exogenous factors, such as brain-derived neurotrophic factor, as well as modulating the expression of endogenous genes, such as sirtuin 1 (SIRT1), can activate Akt to exert a protective effect against DOXO-induced cardiomyopathy $(17,41)$. The PI3K-Akt signaling pathway has been revealed to exert an anti-senescent effect on MSCs under hypoxic conditions, and MIF can activate this pathway (13). The results of the current study demonstrated that MIF activated the Akt pathway and reduced the DOXO-induced senescence of MSCs, as indicated by the increased proliferation, viability, paracrine effects, telomere length and telomerase activity observed following MIF treatment. By contrast, silencing Akt using siRNA attenuated the anti-senescent effect of MIF, suggesting that MSC rejuvenation by MIF is dependent upon the PI3K-Akt signaling pathway.

Oxidative stress is closely associated with the induction of cellular senescence (42). This is accompanied by ROS generation, through increased oxidative enzyme activity and decreased antioxidative enzyme activity (43). DOXO has been associated with cellular DNA damage and endoplasmic reticulum stress, as well as inducing cellular senescence during cardiomyopathy through oxidative stress $(7,8,44)$. Previous studies have demonstrated that MIF is an effective antioxidant agent (45), which by modulating oxidative stress promotes the survival of MSC under hypoxia/serum deprivation conditions (15). Consistent with these results, the present study identified that DOXO induced oxidative stress, increased the generation of ROS and MDA, and decreased the activity of SOD. Conversely, MIF had an antioxidant effect on the DOXO-treated MSCs. Previous study has revealed that by activating Akt-dependent pro-survival and antiapoptotic signaling, SIRT1 enhances oxidative stress defenses, and prevents the senescence and growth arrest of human cardiac progenitor cells treated with DOXO (17). The results of the current study indicated that MIF reduced DOXO-induced oxidative stress through the PI3K-Akt signaling pathway, which was supported by the fact that silencing Akt abolished the antioxidative effects of MIF.

In conclusion, the present study demonstrated that DOXO treatment induced MSC senescence and that pretreatment with MIF could rejuvenate senescent MSCs. The results of the present study suggest that MIF can rescue MSCs from DOXO-induced senescence via regulation of oxidative stress through the PI3K-Akt signaling pathway. These findings highlight potentially novel therapeutic strategies for rejuvenating and protecting MSCs from injury induced by DOXO, and provide a mechanistic understanding for the clinical exploitation of MIF and MSCs in cardiac regeneration therapies for DOXO-induced cardiomyopathy.

\section{Acknowledgements}

The present study was supported by the National Natural Science Foundation of China (grant no. 81500261 to M.H.), the Science and Technology Planning Project of Wenzhou (grant no. Y20160125 to M.H.) and the Medical 
Science and Technology Project of Zhejiang Province (grant no. 2018236627 to M.H.).

\section{References}

1. Rochette L, Guenancia C, Gudjoncik A, Hachet O, Zeller M, Cottin Y and Vergely C: Anthracyclines/trastuzumab: New aspects of cardiotoxicity and molecular mechanisms. Trends Pharmacol Sci 36: 326-348, 2015.

2. Butany J, Ahn E and Luk A: Drug-related cardiac pathology. J Clin Pathol 62: 1074-1084, 2009

3. Piegari E, De Angelis A, Cappetta D, Russo R, Esposito G, Costantino S, Graiani G, Frati C, Prezioso L, Berrino L, et al: Doxorubicin induces senescence and impairs function of human cardiac progenitor cells. Basic Res Cardiol 108: 334, 2013.

4. Du WW, Yang W, Chen Y, Wu ZK, Foster FS, Yang Z, Li X and Yang BB: Foxo3 circular RNA promotes cardiac senescence by modulating multiple factors associated with stress and senescence responses. Eur Heart J 38: 1402-1412, 2017.

5. Oliveira MS, Carvalho JL, Campos ACDA, Gomes DA, de Goes AM and Melo MM: Doxorubicin has in vivo toxicological effects on ex vivo cultured mesenchymal stem cells. Toxicol Lett 224: 380-386, 2014.

6. Ezquer F, Gutiérrez J, Ezquer M, Caglevic C, Salgado HC and Calligaris SD: Mesenchymal stem cell therapy for doxorubicin cardiomyopathy: Hopes and fears. Stem Cell Res Ther 6: 116, 2015.

7. Yang F, Chen H, Liu Y, Yin K, Wang Y, Li X, Wang G, Wang S, Tan X, Xu C, et al: Doxorubicin caused apoptosis of mesenchymal stem cells via p38, JNK and p53 pathway. Cell Physiol Biochem 32: 1072-1082, 2013.

8. Capasso S, Alessio N, Squillaro T, Di Bernardo G, Melone MA, Cipollaro M, Peluso $\mathrm{G}$ and Galderisi U: Changes in autophagy, proteasome activity and metabolism to determine a specific signature for acute and chronic senescent mesenchymal stromal cells. Oncotarget 6: 39457-39468, 2015.

9. Miller EJ, Li J, Leng L, McDonald C, Atsumi T, Bucala R and Young LH: Macrophage migration inhibitory factor stimulates AMP-activated protein kinase in the ischaemic heart. Nature 451: 578-582, 2008

10. Xu X, Pacheco BD, Leng L, Bucala R and Ren J: Macrophage migration inhibitory factor plays a permissive role in the maintenance of cardiac contractile function under starvation through regulation of autophagy. Cardiovasc Res 99: 412-421, 2013

11. Wang J, Tong C, Yan X, Yeung E, Gandavadi S, Hare AA, Du X, Chen Y, Xiong H, Ma C, et al: Limiting cardiac ischemic injury by pharmacological augmentation of macrophage migration inhibitory factor-AMP-activated protein kinase signal transduction. Circulation 128: 225-236, 2013

12. Ohta S, Misawa A, Fukaya R, Inoue S, Kanemura Y, Okano H, Kawakami Y and Toda M: Macrophage migration inhibitory factor (MIF) promotes cell survival and proliferation of neural stem/progenitor cells. J Cell Sci 125: 3210-3220, 2012.

13. Palumbo S, Tsai TL and Li WJ: Macrophage migration inhibitory factor regulates AKT signaling in hypoxic culture to modulate senescence of human mesenchymal stem cells. Stem Cells Dev 23: 852-865, 2014.

14. Xia W, Zhang F, Xie C, Jiang $M$ and Hou M: Macrophage migration inhibitory factor confers resistance to senescence through CD74-dependent AMPK-FOXO3a signaling in mesenchymal stem cells. Stem Cell Res Ther 6: 82, 2015.

15. Xia W, Xie C, Jiang M and Hou M: Improved survival of mesenchymal stem cells by macrophage migration inhibitory factor. Mol Cell Biochem 404: 11-24, 2015.

16. Okada M, Kim HW, Matsu-ura K, Wang YG, Xu M and Ashraf M: Abrogation of age-induced MicroRNA-195 rejuvenates the senescent mesenchymal stem cells by reactivating telomerase. Stem Cells 34: 148-159, 2016.

17. De Angelis A, Piegari E, Cappetta D, Russo R, Esposito G, Ciuffreda LP, Ferraiolo FA, Frati C, Fagnoni F, Berrino L, et al: SIRT1 activation rescues doxorubicin-induced loss of functional competence of human cardiac progenitor cells. Int J Cardiol 189: 30-44, 2015 .

18. Kuilman T, Michaloglou C, Mooi WJ and Peeper DS: The essence of senescence. Genes Dev 24: 2463-2479, 2010.

19. De Falco E, Carnevale R, Pagano F, Chimenti I, Fianchini L, Bordin A, Siciliano C, Monticolo R, Equitani F, Carrizzo A, et al: Role of NOX2 in mediating doxorubicin-induced senescence in human endothelial progenitor cells. Mech Ageing Dev 159: 37-43, 2016.
20. Ghosh AK, Rai R, Park KE, Eren M, Miyata T, Wilsbacher LD and Vaughan DE: A small molecule inhibitor of PAI-1 protects against doxorubicin-induced cellular senescence: Molecular basis. Oncotarget 7: 72443-72457, 2016.

21. Ho JH, Chen YF, Ma WH, Tseng TC, Chen MH and Lee OK Cell contact accelerates replicative senescence of human mesenchymal stem cells independent of telomere shortening and p53 activation: roles of Ras and oxidative stress. Cell Transplant 20 : 1209-1220, 2011

22. Liu WH, Liu JJ, Wu J, Zhang LL, Liu F, Yin L, Zhang MM and Yu B: Novel mechanism of inhibition of dendritic cells maturation by mesenchymal stem cells via interleukin-10 and the JAK1/STAT3 signaling pathway. PLoS One 8: e55487, 2013.

23. Hou M, Cui J, Liu J, Liu F, Jiang R, Liu K, Wang Y, Yin L, Liu W and Yu B: Angiopoietin-like 4 confers resistance to hypoxia/ serum deprivation-induced apoptosis through PI3K/Akt and ERK1/2 signaling pathways in mesenchymal stem cells. PLoS One 9: e85808, 2014.

24. Livak KJ and Schmittgen TD: Analysis of relative gene expression data using real-time quantitative PCR and the 2(-Delta Delta C(T)) uethod. Methods 25: 402-408, 2001.

25. Crepin T, Carron C, Roubiou C, Gaugler B, Gaiffe E, SimulaFaivre D, Ferrand C, Tiberghien P, Chalopin JM, Moulin B, et al: ATG-induced accelerated immune senescence: Clinical implications in renal transplant recipients. Am J Transplant 15: 1028-1038, 2015

26. Bray F, Jemal A, Grey N, Ferlay J and Forman D: Global cancer transitions according to the Human Development Index (2008-2030): A population-based study. Lancet Oncol 13: 790-801, 2012.

27. Horie T, Ono K, Nishi H, Nagao K, Kinoshita M, Watanabe S, Kuwabara Y,Nakashima Y, Takanabe-Mori R, NishiE, et al: Acute doxorubicin cardiotoxicity is associated with miR-146a-induced inhibition of the neuregulin-ErbB pathway. Cardiovasc Res 87: 656-664, 2010

28. Volkova M and Russell R III: Anthracycline cardiotoxicity: Prevalence, pathogenesis and treatment. Curr Cardiol Rev 7: 214-220, 2011.

29. Franco VI,Henkel JM, Miller TL and Lipshultz SE: Cardiovascular effects in childhood cancer survivors treated with anthracyclines. Cardiol Res Pract 2011: 134679, 2011.

30. Fan GC, Zhou X, Wang X, Song G, Qian J, Nicolaou P, Chen G, Ren $X$ and Kranias EG: Heat shock protein 20 interacting with phosphorylated Akt reduces doxorubicin-triggered oxidative stress and cardiotoxicity. Circ Res 103: 1270-1279, 2008.

31. Fisher PW, Salloum F, Das A, Hyder H and Kukreja RC: Phosphodiesterase-5 inhibition with sildenafil attenuates cardiomyocyte apoptosis and left ventricular dysfunction in a chronic model of doxorubicin cardiotoxicity. Circulation 111: 1601-1610, 2005.

32. Piegari E, Russo R, Cappetta D, Esposito G, Urbanek K, Dell'Aversana C, Altucci L, Berrino L, Rossi F and De Angelis A: MicroRNA-34a regulates doxorubicin-induced cardiotoxicity in rat. Oncotarget 7: 62312-62326, 2016.

33. Zhang Y, Liang X, Liao S, Wang W, Wang J, Li X, Ding Y, Liang Y, Gao F, Yang M, et al: Potent paracrine effects of human induced pluripotent stem cell-derived mesenchymal atem xells attenuate doxorubicin-induced cardiomyopathy. Sci Rep 5: $11235,2015$.

34. Mao C, Hou X, Wang B, Chi J, Jiang Y, Zhang C and Li Z: Intramuscular injection of human umbilical cord-derived mesenchymal stem cells improves cardiac function in dilated cardiomyopathy rats. Stem Cell Res Ther 8: 18, 2017.

35. Zhang Z, Xu K, Bi Y, Yu G, Wang S, Qi X and Zhong H: Low intensity ultrasound promotes the sensitivity of rat brain glioma to Doxorubicin by down-regulating the expressions of p-glucoprotein and multidrug resistance protein 1 in vitro and in vivo. PLoS One 8: e70685, 2013.

36. Baugh JA, Chitnis S, Donnelly SC, Monteiro J, Lin X, Plant BJ, Wolfe F, Gregersen PK and Bucala R: A functional promoter polymorphism in the macrophage migration inhibitory factor (MIF) gene associated with disease severity in rheumatoid arthritis. Genes Immun 3: 170-176, 2002.

37. Ma H, Wang J, Thomas DP, Tong C, Leng L, Wang W, Merk M, Zierow S, Bernhagen J, Ren J, et al: Impaired macrophage migration inhibitory factor (MIF)-AMPK activation and ischemic recovery in the senescent heart. Circulation 122: 282-292, 2010.

38. Maity A and Koumenis C: HIF and MIF - a nifty way to delay senescence? Genes Dev 20: 3337-3341, 2006. 
39. Ibrahim MX, Sayin VI, Akula MK, Liu M, Fong LG, Young SG and Bergo MO: Targeting isoprenylcysteine methylation ameliorates disease in a mouse model of progeria. Science 340: 1330-1333, 2013

40. Li W, Croce K, Steensma DP, McDermott DF, Ben-Yehuda O and Moslehi J: Vascular and metabolic implications of novel targeted cancer therapies: Focus on kinase inhibitors. J Am Coll Cardiol 66: 1160-1178, 2015

41. Hang P, Zhao J, Sun L, Li M, Han Y, Du Z and Li Y: Brain-derived neurotrophic factor attenuates doxorubicin-induced cardiac dysfunction through activating Akt signalling in rats. J Cell Mol Med 21: 685-696, 2017.

42. Henson SM, Lanna A, Riddell NE, Franzese O, Macaulay R, Griffiths SJ, Puleston DJ, Watson AS, Simon AK, Tooze SA, et al: p38 signaling inhibits mTORC1-independent autophagy in senescent human $\mathrm{CD}^{+} \mathrm{T}$ cells. J Clin Invest 124: 4004-4016, 2014.
43. Finkel T and Holbrook NJ: Oxidants, oxidative stress and the biology of ageing. Nature 408: 239-247, 2000.

44. Lee KH, Cho H, Lee S, Woo JS, Cho BH, Kang JH, Jeong YM, Cheng XW and Kim W: Enhanced-autophagy by exenatide mitigates doxorubicin-induced cardiotoxicity. Int J Cardiol 232: 40-47, 2017.

45. Sobierajski J, Hendgencotta U, Luedike P, Rammos C, Bernhagen J, Kelm M and Rassaf T: Time dependent regulation of MIF by S-nitros(yl)ation reduces apoptosis in myocardial I/R-injury. Eur Heart J 31: S18-S19, 2013. 